\section{Nasenbluten: Heiße Dusche statt Tampon}

In einer Schweizer Studie zeigte eine $50^{\circ} \mathrm{C}$ heiße, dreiminütige „Nasendusche" eine 82\%-ige Erfolgsquote bei schwerem Nasenbluten. Die Spülung erfolgte unter Lokalanästhesie über einen speziellen Ballonkatheter. Das heiße Wasser bewirkt eine Vasodilatation und Schwellung der Nasenschleimhaut. Blutende Gefäße werden dadurch komprimiert. Das Nasenbluten sistiert allgemein innerhalb von zehn Minuten und die Patienten können bereits nach zwei Stunden entlassen werden. Vielen Patienten könnten damit die üblichen, oft schmerzhaften Nasentamponaden erspart bleiben. J Laryngol Otol 2012 126(1): 58

\section{Demenz: SSRI erhöhen Sturzgefahr}

Nicht nur anticholinerge Trizyklika, auch moderne SSRI - inzwischen Mittel erster Wahl bei älteren Menschen mit Depressionen - erhöhen bei Demenzpatienten das Sturzrisiko. Eine Studie mit 248 Patienten erfasste innerhalb eines Jahres die Sturzfrequenz und setzte diese in Bezug zur SSRI-Einahme: Bei voller empfohlener Tagesdosis erhöhte sich die Sturzfrequenz um den Faktor 3. Wurden SSRI in Kombination mit Hypnotika und Sedativa verschrieben, erhöhte sich das Risiko sogar um das Siebenfache. Brit J Clin Pharmac 2012 (online first)

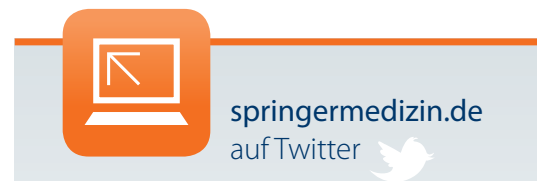

Auch im Winter heißt es: "Lauschen" Sie unserem Zwitschern und werden Sie zum „Follower"! • twitter.com/springermedizin

Intraartikuläre Injektionen

\title{
Hyaluronsäure bei Arthrose? Kochsalz tut's auch!
}

Intraartikuläre Injektionen von Hyaluronsäure richten gegen eine Sprunggelenksarthrose auch nicht mehr aus als Spritzen, die gewöhnliche Kochsalzlösung enthalten.

In einer Studie haben Ärzte aus Newton (USA) 56 Patienten mit Sprunggelenksarthrose in zwei Gruppen eingeteilt. Probanden der Gruppe eins erhielten eine einzelne intraartikuläre Injektion von 2,5 ml niedermolekularer, unvernetzter Hyaluronsäure. Teilnehmern der Gruppe zwei wurden $2,5 \mathrm{ml}$ gewöhnliche Kochsalzlösung gespritzt. Der Behandlungserfolg wurde nach sechs und zwölf Wochen mit dem AOFAS-Score der American Orthopaedic Foot and Ankle Society (0-100 Punkte) bestimmt.

\section{Unterschied nicht signifikant}

$\mathrm{Zu}$ Beginn der Studie unterschieden sich die mittleren Werte in beiden Gruppen nicht signifikant, sie lagen bei rund 65 Punkten. Nach sechs Wochen hatte sich der Score in der Verumgruppe um 4,9 Punkte verbessert, in der Placebogruppe um 0,4 Punkte verschlechtert; die Differenz erwies sich als nicht signifikant. Weitere sechs Wochen später lag der Zugewinn gegenüber dem Ausgangswert für die mit Hyaluronsäure Behandelten immer noch bei 4,9 Punkten, nach der Kochsalzspritze war nun ein Plus von 5,4 Punkten zu verzeichnen. Hyaluronsäure oder Kochsalz - handelte es sich nicht ausgerechnet um Arthrosepatienten, müsste das Fazit lauten: Es ist gehüpft wie gesprungen.

(rb)

J Bone Joint Surg 2012, 94:2

\section{Erfolgreiche Op.}

\section{Probleme mit den Mandeln? Raus damit!}

\section{9 von 20 Patienten profitieren von einer Operation bei Mandelbeschwer- den: Sie sind die Symptome anschlie- ßend ganz oder fast ganz los.}

Die Mandeln zu entfernen scheint tatsächlich die beste Möglichkeit zu sein, um Probleme durch Obstruktionen oder wiederkehrende Entzündungen zu beseitigen, bestätigt eine große schwedische Registerstudie. Bei den 55.000 beteiligten Patienten (zum größten Teil Kinder und Jugendliche) wurden vor der Op. Beschwerden und Indikation erfasst. Sechs Monate später durften die Patienten bzw. deren Eltern den Erfolg des Eingriffs dokumentieren.

\section{Bei drei Viertel verschwinden die Beschwerden komplett}

Die Ergebnisse: 77\% der Operierten berichteten, dass die Symptome verschwunden waren, knapp 20\% gaben an, dass sie fast weg waren. Nur 2,9\% der Patienten waren der Auffassung, dass die Op. nichts gebracht hatte.

Den besten Erfolg brachte dabei eine Kombination von Tonsillektomie plus Adenoidektomie: $80 \%$ der Patienten waren komplett und $17,3 \%$ fast beschwerdefrei. Unterschiede beim Erfolg zwischen ambulant und stationär behandelten Patienten gab es praktisch nicht.

(mut) 